\title{
Complete genomic sequence of the Vibrio alginolyticus bacteriophage Vp670 and characterization of the lysis-related genes, cWlQ and holA
}

Peng Luo ${ }^{1,2}$, Long Yun ${ }^{1,3}$, Yingying $\mathrm{Li}^{1,3}$, Yushun Tian ${ }^{1,3}$, Qiuting Liu ${ }^{1,3}$, Wen Huang ${ }^{1,2^{*}}$ and Chaoqun Hu${ }^{1,2^{*}}$

\begin{abstract}
Background: Biocontrol of bacterial pathogens by bacteriophages (phages) represents a promising strategy. Vibrio alginolyticus, a gram-negative bacterium, is a notorious pathogen responsible for the loss of economically important farmed marine animals. To date, few $V$. alginolyticus phages have been successfully isolated, and only three complete genome sequences of them have been released. The limited available phage resources and poor genomic data hamper research on $V$. alginolyticus phages and their applications for the biocontrol of $V$. alginolyticus.

Results: We isolated a phage, Vp670, against the V. alginolyticus strain E06333 and obtained its full genomic sequence. It contains 43,121 nucleotides with a GC content of 43.4\%, and codes for 49 predicted open reading frames. Observation by electron microscope combined with phylogenetic analysis of DNA polymerase indicates that Vp670 belongs to the subfamily Autographivirinae in the family Podoviridae. orf3 (designated holA) and orf8 (designated cw/Q) are predicted to encode a holin (HolA) and an endolysin (CWIQ), respectively. Expression of holA alone or coexpression of holA and $c$ WIQ from within arrested the growth of Escherichia coli and V. alginolyticus while the expression of cw/Q alone had no effect on the growth of them. Further observation by transmission electron microscopy revealed that the expression of holA vanished the outer membrane and caused the release of cellular contents of $\mathrm{V}$. alginolyticus and the coexpression of holA and $C W / Q$ directly burst the cells and caused a more drastic release of cellular contents. Expression of CWIQ alone in V. alginolyticus did not cause cytomorphological changes.
\end{abstract}

Conclusions: Phage Vp670 is a V. alginolyticus phage belonging to the family of Podoviridae. The genome of Vp670 contains a two-component lysis module, which is comprised of holA and $\mathrm{CW} / \mathrm{Q}$. holA is predicted to encode for the holin protein, HolA, and $c W / Q$ is predicted to encode for the endolysin protein, $\mathrm{Cw}$ Q. Both holA and cwlQ likely play important roles during the release of phage progeny.

Keywords: Vibrio alginolyticus, Bacteriophage, Holin, Endolysin

\section{Background}

Vibrio alginolyticus, a gram-negative bacterium, is an opportunistic pathogen to marine animals and human beings. It has been reported to be an important pathogen responsible for economic losses in several aquatic species [1]. Traditionally, $V$. alginolyticus and other pathogenic Vibrio

\footnotetext{
*Correspondence: huchaoqun62@163.com; huangwen549@126.com 'CAS Key Laboratory of Tropical Marine Bio-resources and Ecology (LMB), Guangdong Provincial Key Laboratory of Applied Marine Biology (LAMB), South China Sea Institute of Oceanology, Chinese Academy of Sciences, Guangzhou 501301, People's Republic of China

Full list of author information is available at the end of the article
}

species are controlled using antibiotics; however, this method is becoming less and less effective due to the emergence of antibiotic-resistant strains, and environmental and ecological problems related to widespread antibiotic use [1, 2]. It is estimated that most bacteria (90\%) isolated from marine environments are resistant to more than one antibiotic and that $20 \%$ of them are resistant to at least five antibiotics [3, 4]. Antibiotics abuse is known to accelerate the emergence of multi-drug-resistant bacteria. Therefore, it is urgent to explore new ways to prevent infection by pathogenic $V$. alginolyticus and other Vibrio species.

(c) The Author(s). 2018 Open Access This article is distributed under the terms of the Creative Commons Attribution 4.0 International License (http://creativecommons.org/licenses/by/4.0/), which permits unrestricted use, distribution, and 
Bacteriophages (phages) belong to a category of viruses that infect and lyse bacteria and are natural and abundant in the marine environment. Phages play important roles in, for example, structuring bacterial diversity and succession in the ocean, promoting biogeochemical cycling of elements, and driving horizontal gene transfer [5, 6]. In recent years, phages have gained much attention as they have been found to have some advantages over antibiotics in terms of controlling bacterial infection [7-9]. Antibiotics persist in the environment while phages proliferate only when a specific bacterial host is present. Therefore, unlike antibiotics, phages do not disturb the normal flora in animals and humans [7-9] and theoretically do not cause the diffusion of drug-resistance genes. Endolysins, lytic enzymes of bacteriophages, are proteins synthesized at the end of the lytic cycle, and they destroy the cell wall peptidoglycan (PG) to facilitate the release of viral progeny [10]. Both phages and phage endolysins are alternative ways to prevent pathogens, especially those with multi-drug resistance. Using purified endolysins may have certain advantages over whole phages $[11,12]$. One significant advantage of endolysins is that, unlike antibiotics and whole phages there have been no reports of the development of bacterial resistance $[11,12]$.

To date, few $V$. alginolyticus phages have been successfully isolated $[1,13,14]$, and only three complete genome sequences of their phages have been released [13]. The limited available phage resources and poor genomic data hamper research on $V$. alginolyticus phages and their use in pathogen biocontrol. In the present study, we sequenced the $V$. alginolyticus phage Vp670 that was isolated from marine water (Shenzhen, China) and performed bioinformatic analysis. The functions of the lysis-related genes, $c w l Q$ and holA, were examined by the expression of each gene or fused genes in Escherichia coli and the host of the phage, $V$. alginolyticus. This study will, to some degree, create a path to the biocontrol of $V$. alginolyticus.

\section{Methods}

\section{Bacterial strains and growth conditions}

$V$. alginolyticus E06333 was isolated from the ulcer of a diseased and cage-cultured fish (Epinephelus daemelii) in Zhanjiang (Guangdong Province, China) in 2006. The ulcer of the fish was externally disinfected with $75 \%$ ethanol and was sampled with a cotton swab. Then the fish were provisionally cultured with a tank with seawater until it died. The animal experiment and sample collection were performed in accordance with the guidelines according to the Department of Scientific Research and Planning of South China Sea Institute of Oceanology, Chinese Academy of Sciences. Strain E06333 is resistance to ampicillin and sulfamethoxazole. $V$. alginolyticus E06333, E. coli DH5 $\alpha$ and their derivative strains (Additional file 1: Table S1) were cultured in Luria-Bertani (LB) broth or on an LB agar plate at $30{ }^{\circ} \mathrm{C}$.

\section{Isolation and purification of phage $\mathrm{Vp} 670$}

The isolation and purification of phage Vp670 was performed as described previously, with minor modifications [15]. V. alginolyticus E06333 was cultured overnight as an indicator host for a plaque assay. Sewage water samples obtained from Dayawan, Yangjiang, and Zhanjiang in Guangdong, China were centrifuged at 10,000 $\mathrm{g}$ for $10 \mathrm{~min}$, and then the supernatants were filtered using a sterile $0.45-\mu \mathrm{m}$ filter. The filtrates were mixed with the same volume of $2 \times$ LB broth and then were added with 1\% overnight culture of E06333 cells followed by incubation at $30{ }^{\circ} \mathrm{C}$ for $12 \mathrm{~h}$. The lytic culture samples (which remained transparent) were centrifuged, the supernatant was pipetted out, and was then filtered through a sterile $0.22-\mu \mathrm{m}$ filter. The filtrate was used to isolate phages through the double-plate method [15]. A single plaque was picked out of the plates and diluted in SM buffer composed of $0.05 \mathrm{M}$ Tris- $\mathrm{HCl}(\mathrm{pH} 7.5), 0.1 \mathrm{M} \mathrm{NaCl}$, $10 \mathrm{mM} \mathrm{MgSO}_{4}$, and $1 \%$ gelatin for subculture and purification.

\section{Morphological observation by transmission electron microscopy}

The morphology of phage Vp670 and the bacterial cells were observed with a Hitachi transmission electron microscope (TEM) after they had been negatively stained with $2 \%(W / V)$ phosphotungstic acid according to the method previously described [16].

\section{The replication parameters of phage Vp670}

One-step growth curve for phage Vp670 was analyzed as previously described $[17,18]$ to determine the replication characteristics of the phage. In brief, an overnight-grown culture of strain E06333 at $30{ }^{\circ} \mathrm{C}$ was inoculated into fresh LB at a ratio of $1 \%$ and grown until the optical density (OD, $600 \mathrm{~nm})$ reached at $1.0(\sim 3 \times$ $10^{8} \mathrm{CFU} / \mathrm{ml}$ ). The bacterial culture was 10 -fold diluted to $\sim 3 \times 10^{7} \mathrm{CFU} / \mathrm{ml}$ and then $500-\mu l$ diluted culture was mixed with the same volume of phage Vp670 solution (Multiplicity of Infection, MOI = 1). The bacteria/phage mixture $(1 \mathrm{ml})$ was added to $19 \mathrm{ml}$ of LB broth. $100-\mu \mathrm{l}$ aliquots from each sample were collected every $10 \mathrm{~min}$ for the first $60 \mathrm{~min}$, and at $90 \mathrm{~min}, 120 \mathrm{~min}, 180 \mathrm{~min}$, and $240 \mathrm{~min}$. The samples were immediately diluted as needed and then incubated on double-layer plates to obtain phage plaques and determine the phage titers. The burst size (Bs) was calculated as $\mathrm{Bs}=\mathrm{Pt} / \mathrm{P0}$, where $\mathrm{Pt}$ is the maximum phage titer at the plateau phase and $\mathrm{PO}$ is the initial infective titer $[17,18]$. The experiments contained three replicates. 


\section{Adsorption rate of phage $\mathrm{Vp} 670$}

The phage adsorption rate assay was performed as previously described, with some minor modifications [19]. The host E06333 cells were cultured overnight. LB broth and $1 \%$ overnight-grown $V$. alginolyticus were incubated at $30{ }^{\circ} \mathrm{C}$ until the optical density (OD, at $\left.600 \mathrm{~nm}\right)$ of this culture reached 1.0. Five hundred microliters of the culture $(\mathrm{OD}=1.0)$ was mixed with same volume of phage Vp670 solution $(\mathrm{MOI}=0.1)$ and then suspended into 19-ml LB broth. This point was regarded as the start time point for sampling. A 1-ml sample was taken every $2 \mathrm{~min}$ until $10 \mathrm{~min}$ and immediately centrifuged at $8000 \mathrm{rpm}$ for $2 \mathrm{~min}$ to separate the bacteria from the suspension composed of free phage. The supernatant was discarded, and then the pellet was resuspended, properly diluted and plated for the phage titer.

\section{Phage genome extraction and genomic sequencing}

After centrifugation of the phage lysate at $10,000 \times \mathrm{g}$ for $10 \mathrm{~min}$, the supernatant was filtered through a $0.45-\mu \mathrm{m}$ filter. Then, the filtrate was used for genome extraction using the Phage Genome Extraction Kit (KnoGen, China) according to the manufacturer's instructions. The genomic DNA was sequenced by Suzhou GENEWIZ Biotechnology Co., LTD using an Illumina Miseq to obtain the complete genome sequence of phage Vp670.

\section{Bioinformatic analysis of phage genome}

Gene prediction and functional annotation were conducted using the RAST server (http://rast.nmpdr.org/) followed by correction with BLAST searches. SMART online (http://smart.embl-heidelberg.de//) was used to predict putative conserved domains. Signal peptides and transmembrane domains were predicted using SignalP 4.0 Server (http://www.cbs.dtu.dk/services/SignalP/) and TMHMM v2.0 Server (http://www.cbs.dtu.dk/services/ TMHMM/), respectively. A hydrophilicity plot of predicted proteins was produced using the ProtScale (https://web.expasy.org/protscale/). To determine the taxonomic status of bacteriophage $\mathrm{Vp670}$, a phylogenetic tree was constructed based on DNA polymerase (DNAP) [20]. The sequences of DNAP from different members of the family Podoviridae were retrieved from GenBank. The sequences were aligned for the construction of a phylogenetic tree using MEGA 6.0 based on the neighbor-joining method. The GenBank accession numbers of these phages are listed in (Additional file 2: Table S2).

\section{Cloning and expression of $h o l A, c w I Q$, and holA-cwlQ in Escherichia coli and $V$. alginolyticus}

The complete holA, $c w l Q$, and holA-cwlQ genes were amplified using PrimSTAR Max DNA Polymerase (Takara, China). The holA-cwlQ gene fusion was constructed by overlap extension PCR. To avoid digestion steps during the expression vector construction, the vector fragment was amplified using pBAD18-Kan as template and then recombinated with the previously amplified $h o l A, c w l Q$, and holA-cwlQ genes, respectively. The resulting plasmids were transformed into competent $E$. coli DH5 $\alpha$ cells (TaKaRa, China) according to the manufacturer's instructions. The pBAD18-holA, pBAD18-cwlQ, and pBAD18-holA-cwlQ plasmids were transferred into $V$. alginolyticus by electroporation according to a previously published method [21]. All the primers used in this study were listed in (Additional file 3: Table S3).

The bacterial cells containing the above-mentioned plasmids were cultured (with kanamycin $[50 \mu \mathrm{g} / \mathrm{ml}]$ and $0.3 \%$ of $\mathrm{D}$-glucose) until the $\mathrm{OD}_{600 \mathrm{~nm}}$ reached at about 0.6. The cells were centrifuged and resuspended with fresh LB medium. The concentration of the resuspended cells were adjusted to $\mathrm{OD}_{600 \mathrm{~nm}}=0.5$, and then were diluted by $10^{6} .0 .1 \mathrm{ml}$ of each diluted culture was spread on an LB agar plate containing kanamycin and L-arabinose $(0.2 \%)$. Three independent replicates of the experiment were performed. Each replicate sample had three independent dilutions and numbers of clones on the plates. In addition, the resuspended cultures of $V$. alginolyticus were supplemented with kanamycin and L-arabinose and incubated for $30 \mathrm{~min}$ at $30{ }^{\circ} \mathrm{C}$ and negatively stained followed by observation using TEM.

\section{Results}

\section{Bacteriophage isolation and morphology}

The diameter of the plaques produced by phage Vp670 was approximately $4-5 \mathrm{~mm}$ after overnight incubation (Additional file 4: Figure S1). Observation by TEM showed that, compared to normal $V$. alginolyticus E06333 cells (Fig. 1a), the cell walls of the infected cells was destroyed, and the outer membrane (OM) layer of the infected cells vanished (Fig. 1b). The infected host cells were filled with phage particles (Fig. 1b). Phage Vp670 has an icosahedral head with a diameter of approximately $55 \mathrm{~nm}$ and a short tail approximately $15 \mathrm{~nm}$ in length (Fig. 1c and d), which indicated that Vp670 may be a member of the family Podoviridae.

\section{The burst size, latent period, and phage adsorption rate}

Figure $2 \mathrm{a}$ shows the one-step growth curve of phage Vp670. The latent period lasts for the first $30 \mathrm{~min}$, in which the increase of the titer of phage Vp670 was very slow. Then, there was a rapid increase of plaque forming units (PFU), lasting for $30 \mathrm{~min}$ and a plateau phase started at $60 \mathrm{~min}$ after the infection. The burst size of Vp670 is approximately $84 \mathrm{PFU} /$ infected cell at an MOI of 1 at $30{ }^{\circ} \mathrm{C}$, in which the phages were allowed to propagate in a one-step infection under the assumption that every bacterial cell was infected by one phage. In $10 \mathrm{~min}$ of tested, the phages adsorbed to host cells 

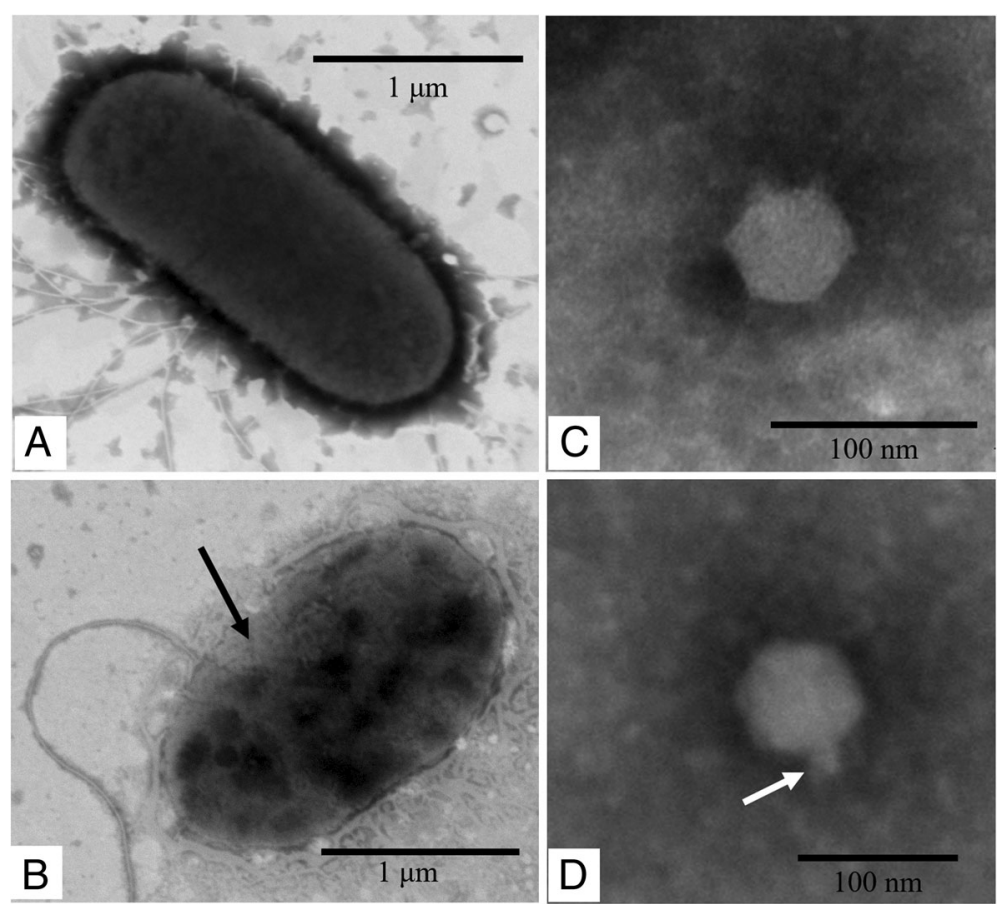

Fig. 1 Electron micrograph of $V$. alginolyticus and phage Vp670. a A normal $V$. alginolyticus cell. b An infected $V$. alginolyticus cell on the verge of lysis. Numerous phage particles (shown by a black arrow) have been assembled within the cell. c The morphology of phage Vp670. $\mathbf{d}$ The short tail of phage Vp670 is shown by the black arrow

accounted for $3 \%$ of total phages 2 min after the starting point, and the phage adsorption rate reached $50 \%$ in another 4 min (Fig. 2b).

\section{Genome and comparative genomics of phage Vp670}

Using Miseq sequencing, we obtained the complete genomic sequence of bacteriophage $\mathrm{Vp} 670$, which contains 43,121 nucleotides with a GC content of $43.4 \%$. The Vp670 sequence was deposited in GenBank under the accession number KY290756. There are 49 predicted
Open Reading Frames (ORFs) in the Vp670 genome (Table 1). No tRNA-encoding genes and no toxin genes were identified. Among 49 predicted ORFs, 24 ORFs were found to be similar to functionally characterized genes in the database whereas 25 ORFs encode for hypothetical proteins with unknown functions. Only one ORF, orf18, was located on the negative strand, and the other 48 ORFs were located on the positive strand (Fig. 3). This arrangement is identical to the arrangement of the genes of the classical T7 phage and
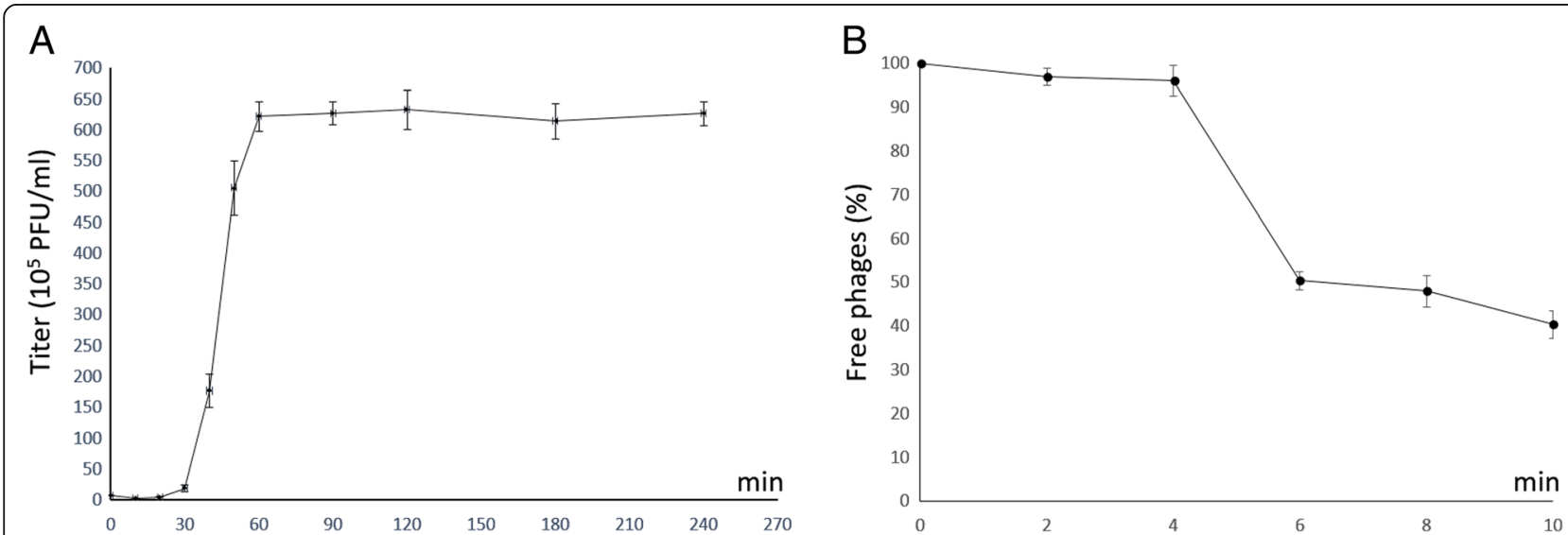

Fig. 2 One-step growth curve and adsorption rate of phage Vp670. a One-step growth curve of phage Vp670. b Adsorption rate of phage Vp670. Triplicate samples were used in each assay 
Table 1 Predicted ORFs of Vibrio alginolyticus phage Vp670a

\begin{tabular}{|c|c|c|c|c|c|}
\hline ORFs & Start & End & Length (bp) & Molecular mass (kD) & Coding for \\
\hline 1 & 114 & 2267 & 2153 & 78.94 & Virion protein \\
\hline 2 & 2385 & 5204 & 2819 & 103.36 & Phage $T 7$ fiber protein \\
\hline 3 & 5213 & 5398 & 185 & 6.78 & Phage holin, HolA \\
\hline 4 & 5400 & 5744 & 344 & 12.61 & Small terminase subunit \\
\hline 5 & 5744 & 7618 & 1874 & 68.71 & Large terminase subunit \\
\hline 8 & 8496 & 8885 & 389 & 14.26 & Endolysin, CwIQ \\
\hline 13 & 11,660 & 11,821 & 161 & 5.90 & Caskin-2/caskin-1 \\
\hline 14 & 11,894 & 12,826 & 932 & 34.17 & Protein kinase \\
\hline 16 & 13,113 & 15,899 & 2786 & 102.15 & DNA-dependent RNA polymerase \\
\hline 23 & 17,580 & 19,676 & 2096 & 76.85 & Hydrolase/Topoisomerase-primase \\
\hline 26 & 20,690 & 23,233 & 2543 & 93.24 & Putative DNA polymerase \\
\hline 33 & 25,816 & 27,036 & 1220 & 44.73 & Phage exonuclease \\
\hline 34 & 27,020 & 27,457 & 437 & 16.02 & Endonuclease VII \\
\hline 35 & 27,457 & 28,473 & 1016 & 37.25 & Calcineurin-like Phosphoesterase \\
\hline 37 & 28,865 & 29,410 & 545 & 19.98 & Phosphomevalonate kinase \\
\hline 38 & 29,410 & 30,420 & 1010 & 37.03 & Adenylation DNA ligase-like protein \\
\hline 41 & 30,888 & 31,352 & 464 & 17.01 & Putative acetyltransferase \\
\hline 43 & 31,537 & 33,165 & 1628 & 59.69 & Phage head to tail connecting protein \\
\hline 44 & 33,165 & 34,064 & 899 & 32.96 & Scaffolding protein \\
\hline 45 & 34,112 & 35,263 & 1151 & 42.20 & Major capsid protein \\
\hline 46 & 35,331 & 35,978 & 647 & 23.72 & Tail protein \\
\hline 47 & 35,981 & 38,602 & 2621 & 96.10 & Tail protein \\
\hline 48 & 38,612 & 39,217 & 605 & 22.18 & Putative internal virion protein $\mathrm{A}$ \\
\hline
\end{tabular}

${ }^{\mathrm{a} A}$ total of 26 ORFs are predicted to encode for hypothetical proteins, which are omitted from the table

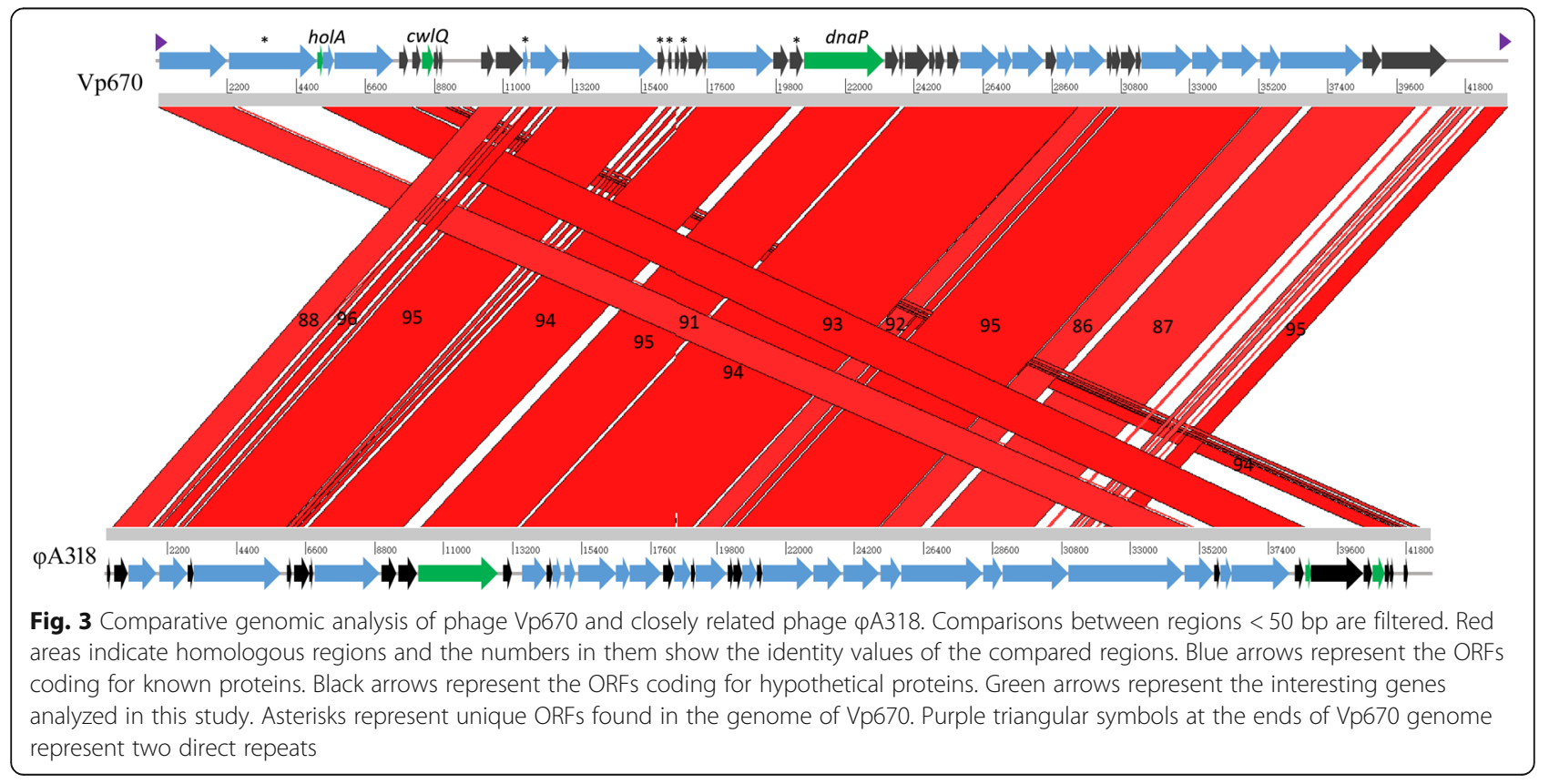


BPP-1-like phage in the Podoviridae family [22]. The backbone of the Vp670 genome is divided into four modules, and each module consisted of several functionally related genes. These modules include the replication and regulation module (orf13, orf14, orf16, orf23, orf 26, orf34, orf35, orf37, and orf38), the lysis module (orf3 and orf8), the packaging module (orf4 and orf5), and the structure module (orf43, orf44, orf45, orf46, orf47, orf1, and $\operatorname{orf} 2$ ).

The whole genomic sequence of Vp670 has $88 \%$ similarity to that of bacteriophage $\phi A 318$ (GenBank: KF322026.1). This similarity was mainly due to two aspects: the genomic collinearity (Fig. 3) and DNAP (Fig. 4), which is generally used as a taxonomic marker [23].

The phylogenetic tree based on DNAP sequences revealed that the subfamily Autographivirinae from that Podoviridae family was grouped into a clade, including Enterobacteria phage T7, Enterobacteria phage SP6, Klebsiella phage KP34, and Pseudomonas phage phiKMV, separated from the members of Picovirinae (Fig. 4). Moreover, V. alginolyticus phage Vp670, Vibrio phage Vc1 and two other $V$. alginolyticus phages ( $\phi$ A318 and AS51) are also included in a smaller clade. The
DNAP sequences of this clade have 99\% similarity with that of Enterobacteria phage SP6, a member of Autographivirinae. In this way, bacteriophage Vp670 was classified as a member of subfamily Autographivirinae.

Despite the $88 \%$ sequence similarity between VP670 and $\phi \mathrm{A} 318$, the genomic content and structure of Vp670 were different from those of $\phi A 318$. The genome of Vp670 was divided into four modules: the replication and regulation module, the lysis module, the packaging module and the structure module. The genes related to regulatory functions are more diverse than those of phage $\phi A 318$. The Vp670 genome also contains six unique genes that are not found in the $\phi A 318$ genome (Fig. 3). There are three obvious genomic rearrangements in Vp670 (nt 1-2395, nt 5212-8090, and nt 8225-9149) compared with $\phi A 318$ (Fig. 3). Three rearrangement regions mainly contain four ORFs that $\mathrm{pu}-$ tatively code for a virion protein, a holin, a terminus, and an endolysin protein. Interestingly, although both Vp670 and $\phi A 318$ are $V$. alginolyticus-infecting phages with high sequence similarity, the Vp670 genome is flanked by a 363-bp terminal redundancy whereas \$A318 lacks an obvious terminal redundancy region

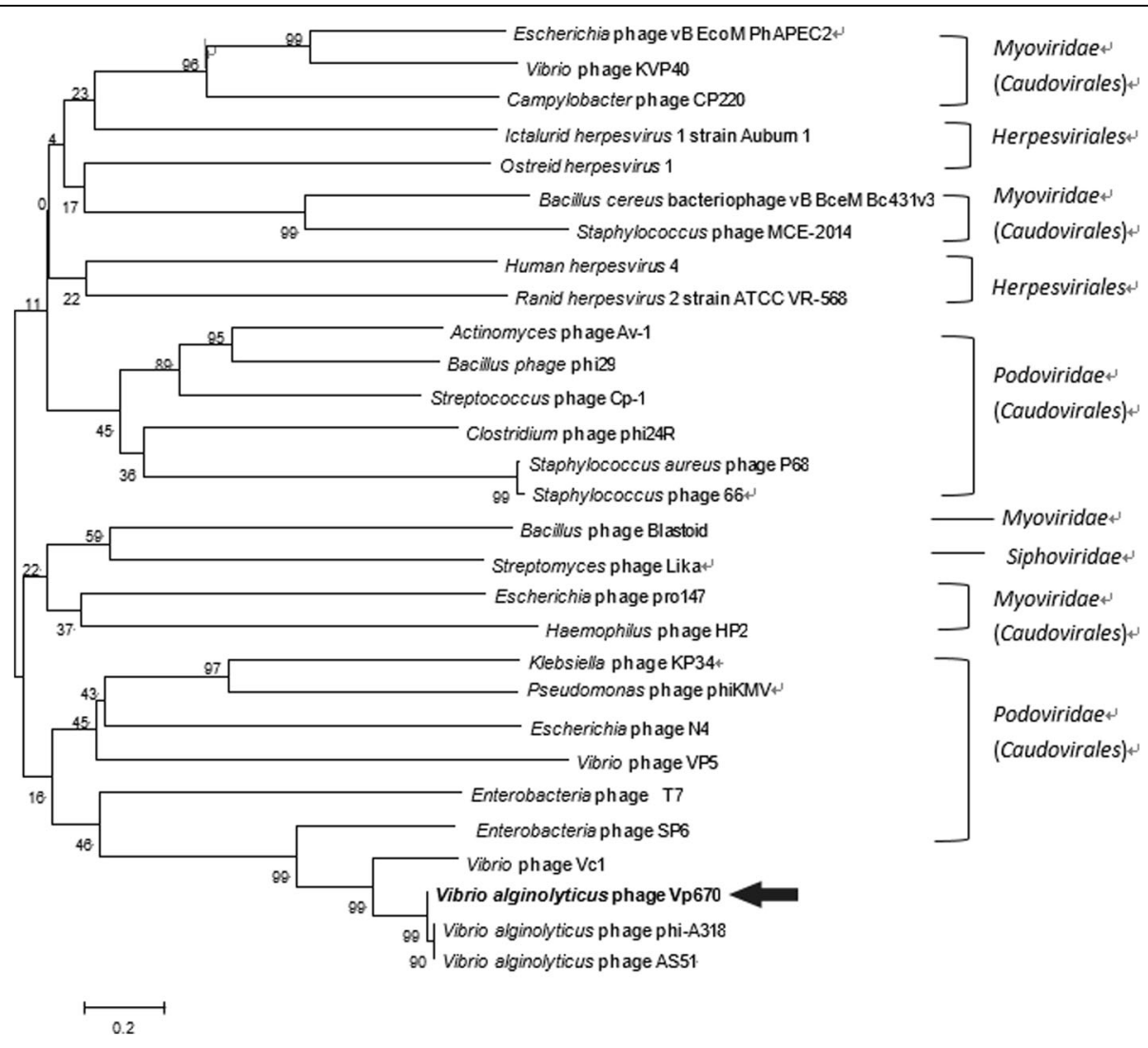

Fig. 4 Phylogenetic tree based on the DNA polymerase sequences of phages in the family of Podoviridae. The tree was constructed using the neighbor-joining method. It shows that the position of V. alginolyticus phage Vp670 (shown by the black arrow) is within the subfamily Autographivirinae, closely related to Enterobacteria phage SP6. The numbers at the nodes indicate the levels of bootstrap support based on data for 1000 replicates. The bar shows $20 \%$ sequence divergence 
(Fig. 3). However, we could not rule out that the lack of an obvious $\phi$ A318 terminal redundancy region was caused by the method of sequencing or assembly. Terminal redundancy is found extensively in phage genomes (e.g., T7, T4, and P1), and it could mean that phages with this feature may adopt the same strategy to replicate genomic DNA.

\section{Lysis-related genes of Vp670}

In phage Vp670, orf8 has 99\% identity with a putative hydrolase gene from $\phi$ A318. BLASTX also showed that the hypothetical product of orf8 has low identity values (37$39 \%$ ) with a series of cell wall hydrolases from a wide variety of sources. Further searches using SMART showed that the predicted protein encoded by orf 8 has a hydrolase- 2 superfamily domain (Pfam 07486). No other ORFs were found to code for an endolysin. Therefore, we speculated that orf8 (designated as $c w l Q$ ) represents a novel endolysin, named CwlQ. orf3 (designated holA) is located upstream of orf8 and encodes a putative phage holin, HolA. HolA was predicted to contain a transmembrane helix (Fig. 5a) with a hydrophilic C-terminal region inside the cytoplasmic membrane (Fig. 5b).

\section{The effect of the expression of holA and $c w / Q$ on E. coli and V. alginolyticus cells}

To explore the function of holA and $c w l Q$, we calculated the number of colonies on LB plates supplemented with $\mathrm{D}$-glucose or L-arabinose. The results indicated that the E. coli (LPN028 and LPN030) and $V$. alginolyticus (LPN041 and LPN043) cells expressing HolA or HolA and CwlQ could not survive under the L-arabinose induction conditions (Fig. $6 \mathrm{a}$ and b). This demonstrated that not only the coexpression of holA and $c w l Q$ kills all the recombinant cells but also the expression of holA alone can kill the induced cells without the participation of induced CwlQ. The expression of endolysin gene alone, $\mathrm{c} w l Q$, has no obvious negative effect on $E$. coli LPN029 (Fig. 6a) or $V$. alginolyticus LPN042 (Fig. 6b).

To further verify the functions of holA and $c w l Q$, the induced $V$. alginolyticus cells LPN040, LPN041, LPN042, and LPN043 were also observed using TEM. LPN040

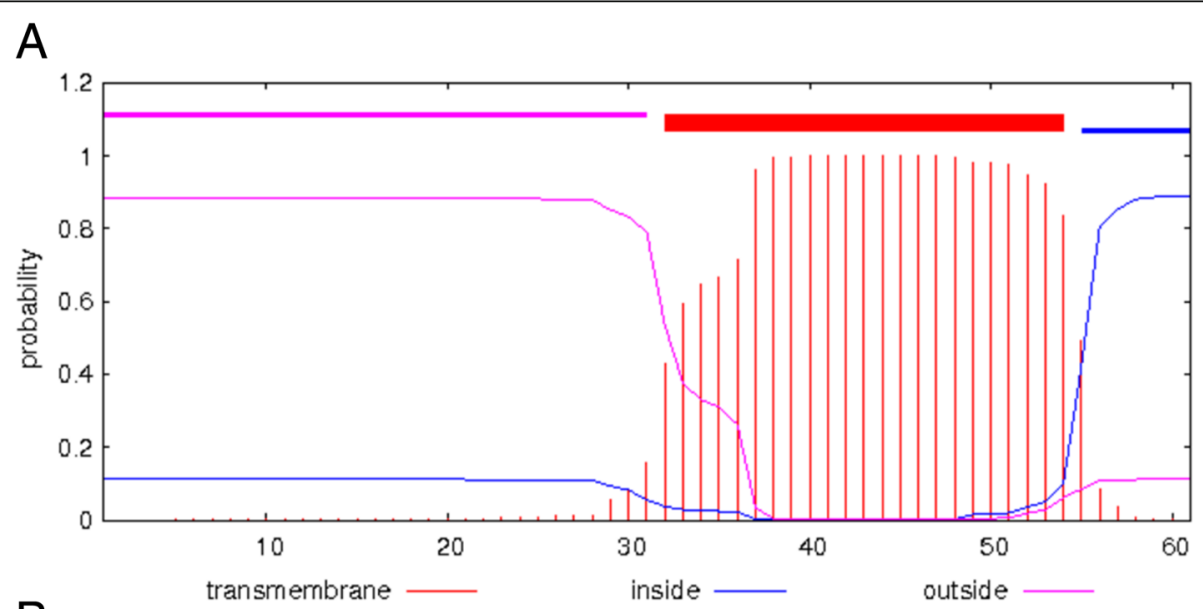

B

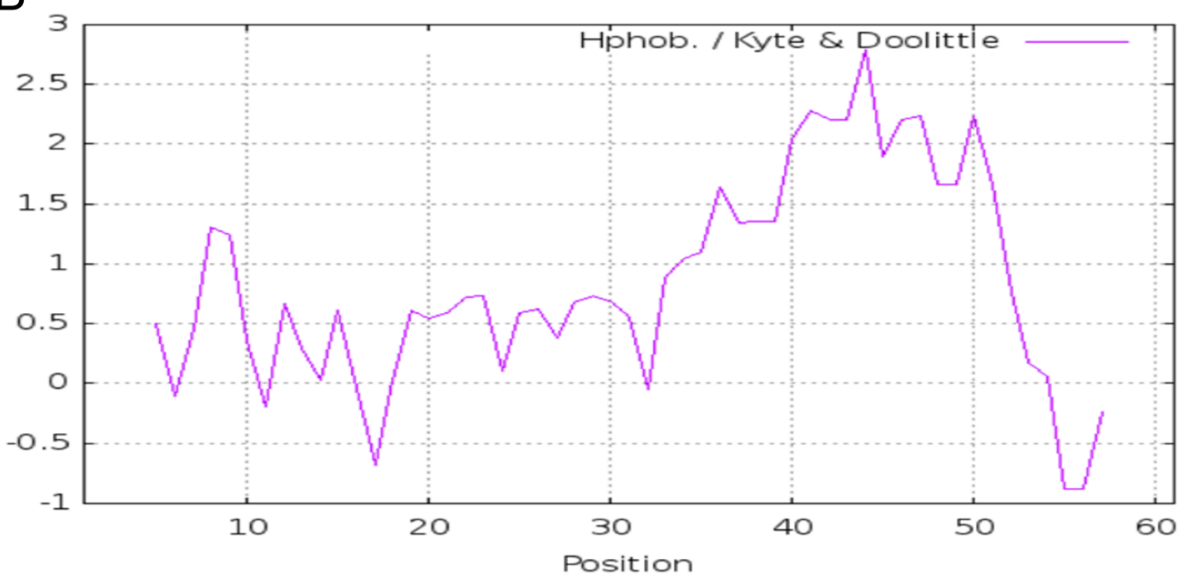

Fig. 5 Structural domain and hydrophilicity prediction of HolA from Vp670. a Transmembrane domain analysis by TMHMM. b Hydrophilicity analysis shows that HolA has a hydrophilic C terminus 

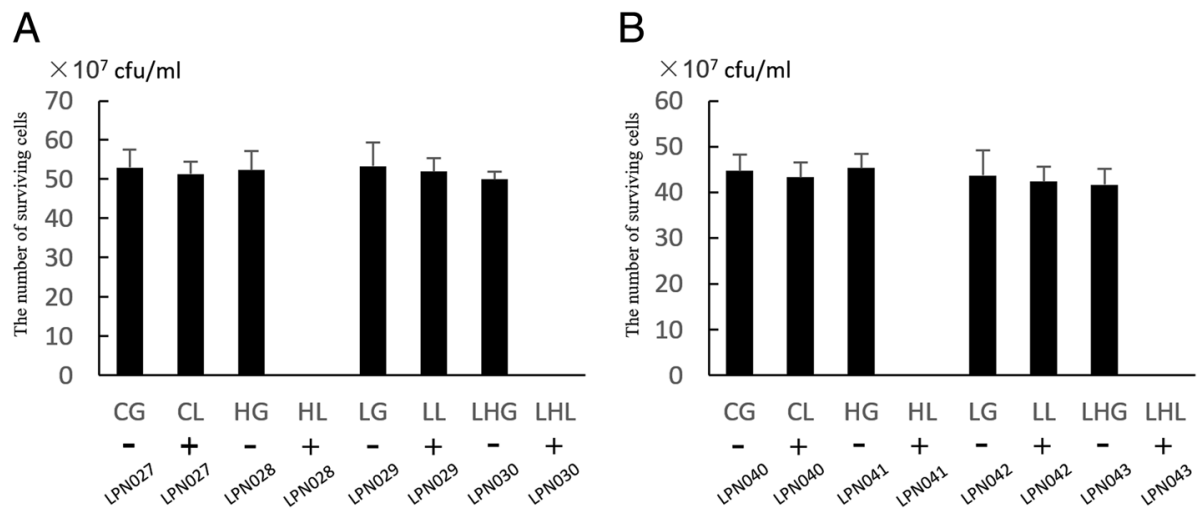

Fig. 6 The impact of the expression of holA and $c w / Q$ on the survival of the host cells. "-" represents the blocked expression of target genes by D-glucose and " + " represents the expression of target genes activated by L-arabinose. a Quantification of the survival of E. coli cells carrying different recombinant plasmids. LPN027 (pBAD18kan), LPN028 (pBAD18-ho/A), LPN029 (pBAD18-cw/Q), and LPN030 (pBAD18-ho/A-cw/Q). b Quantification of the $V$. alginolyticus cells carrying different expression plasmids. LPN040 (pBAD18kan), LPN041 (pBAD18-holA), LPN042 (pBAD18-cw/Q), and LPN043 (pBAD18-holA-cw/Q). Triplicate samples were used in each assay

cells without holA and cwlQ expression and LPN042 cells with $c w l Q$ expression had intact cellular structures (Fig. 7a and Fig. 7d), and there was no obvious morphological difference between them. The OM layer of induced LPN041 cells expressing HolA either disrupted or vanished and their cellular contents were released from some channels in the cell membrane (Fig. 7b), forming some bubbles outside the cell membrane (Fig. 7c). The release of cellular contents resulted in some intracellular light-colored regions (Fig. 7b). However, the coexpression of holA and $c w l Q$ in LPN043 cells caused more serious damage to the structure of the cells than the expression of holA alone in the cells. Staining of whole LPN043 cells with induced HolA and CwlQ revealed that they had become shallow and rough textured. Many particles were also observed inside the cells (Fig. 7e), which represented a larger loss and condensation of cellular contents. Drastic destruction by induced HolA and CwlQ was also confirmed by the observation of a large breach that allowed the release of numerous cellular contents (Fig. 7f). These results verified that the expression of holA destroyed the cellular membrane and killed the induced cells and that coexpression of holA and cwlQ could induce cell bursting and cause more drastic release of cellular contents.

\section{Discussion}

$V$. alginolyticus is a ubiquitous bacterium found in the marine environment. It has been associated with disease in aquatic animals but also in humans, causing tissue damage in the skin, ears and internal organs [24-26]. Like phage therapy against other pathogenic Vibrio species such as $V$. parahaemolyticus [27] and $V$. vulnificus [28], phage therapy also represents a promising strategy for biocontrol of the infection by $V$. alginolyticus [13].
However, until now, few $V$. alginolyticus phages were isolated and subjected to functional genomic analysis. In this study, $V$. alginolyticus phage $\mathrm{Vp} 670$ was isolated and its taxonomy, genomic contents, infection kinemics, and the functions of lysis-related genes were characterized.

Phylogenetic analysis based on DNAP sequences indicated that $\mathrm{Vp} 670$ is closely related to two $V$. alginolyticus phages, $\phi A 318$ and AS51, and belongs to Podoviridae family. Compared with the burst sizes of $\phi A 318$ (72 $\mathrm{PFU} /$ cell) [17] and another $V$. alginolyticus phage фGrn1 (44 PFU/cell) [14], Vp670 had a relatively higher burst size (approximately 84 PFU/infected cell), which may imply that Vp670 has a strong $V$. alginolyticus infection ability.

Phages in Podoviridae family are characterized by an isomorphous icosahedral head and a short noncontractile tail [17]. Most tailed phages cause correctly timed host cell lysis through the consecutive action of a two-component lysis system composed of endolysin and holin $[29,30]$. In present study, orf3 (holA) was predicted to encode a phage holin, HolA, by our BLAST search. The putative HolA contains a transmembrane helix with a hydrophilic C-terminal region inside the cytoplasmic membrane. These features of HolA are similar to the description of common holing characteristics [31], which further strengthened our speculation that holA encodes for a holin. Holins are produced during the late stages of infection, and once a critical concentration is reached, holins form holes in the cytoplasmic membrane by oligomerization, allowing endolysins, which have accumulated in the cytoplasm, to access their PG substrate [32, 33]. Holins trigger the formation of holes that collapse the membrane proton motive force, leading to immediate growth cessation and cell death [30, 34]. In this study, we also observed that not 


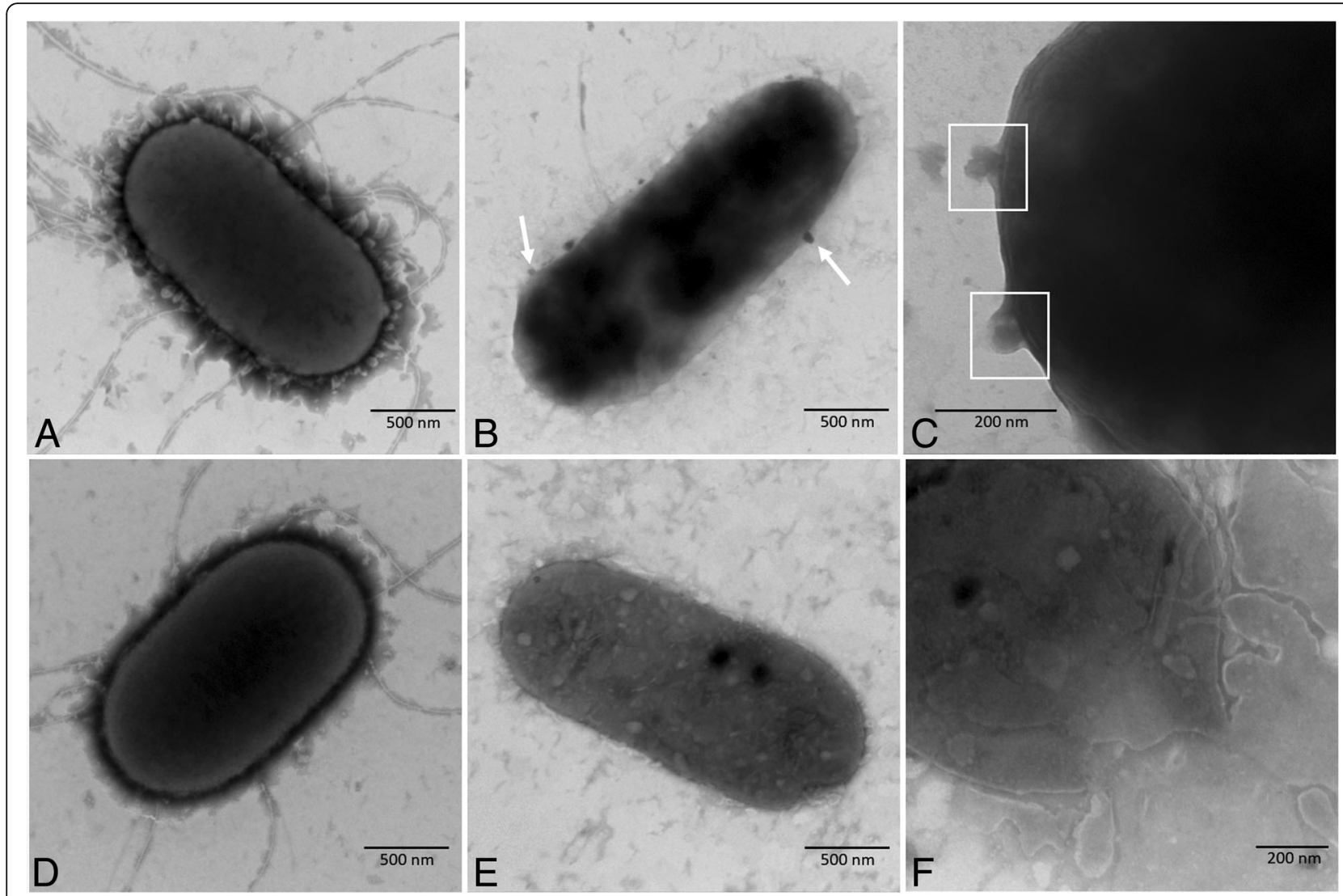

Fig. 7 Electron micrograph of the induced V. alginolyticus cells. a LPN040 cells without the expression of holA or cW/Q. b LPN041 cells with the expression of holA. White arrows show the bubbles on the surfaces of the cells. $\mathbf{c}$ Enlarged image of a LPN041 cell that expresses holA. White boxes indicates two bubbles. $\mathbf{d}$ LPN042 cells with the expression of CWIQ. e LPN043 cells with the expression of holA and cW/Q. $\mathbf{f}$ Enlarged image of a LPN043 cell that expresses holA and CWIQ

only coexpression of holA and cwlQ killed all the recombinant cells but also of expression of holA alone could completely arrest the growth of $E$. coli and $V$. alginolyticus cells. Further TEM observation confirmed that HolA killed the induced $V$. alginolyticus cells through destroying cellular structures and causing the release of cellular contents. Typical holins spontaneously assemble into oligomers, resulting in the formation of nonspecific and very large pores in the cytoplasmic membrane that allow the nonspecific release of endolysins and other proteins $[31,35,36]$. Though the expression of holA had ability to kill and disrupt the integrity of the bacterial cell wall, it could not rapidly cause large breaches on the cell walls and burst the cells without the synergistic effects of the $c w l Q$ expression. Apparently, big breaches or cellular burst facilitate the release of phage progeny particles.

Interestingly, though holA originates from $V$. alginolyticus phage Vp670, the heterogenic expression of holA killed E. coli cells as well. This is not the only such case, and similar phenomena have been observed. Heterogenic expression of holin genes from various sources exhibits toxicity towards $E$. coli cells [31, 37, 38]. It suggests that the integration of holins into cell membranes may lack species specificity [31, 38], and therefore, holA and even other holin genes are potentially valuable for some applications. For instance, holA may be used as a counterselective marker for constructing suicide vectors instead of the unsatisfactory $s a c B$ gene, which is not universal enough for use in the manipulation of bacterial genetics [21]. As holins can form nonspecific tunnels in bacterial cytomembranes [39], holin-based lytic systems have the potential to function as deliver systems, introducing drugs, nucleic acids, and proteins into eukaryotic cells [40].

The predicted CwlQ exhibits typical features of endolysins from phages of gram-negative bacteria: small molecular mass (between 15 and $20 \mathrm{kDa}$ ) and the lack of specific cell wall binding domains $[41,42]$. In this study, coexpression of $c w l Q$ and holA in $V$. alginolyticus thoroughly destroyed bacterial cell walls and burst the cells, causing more drastic damage than the expression of holA alone. This implied that $c w l Q$-encoded CwlQ acts as an endolysin but further verification of its function needs to be performed. 


\section{Conclusions}

Phage Vp670 is a $V$. alginolyticus phage belonging to Podoviridae family. Phage Vp670 could rapidly absorb to the surface of $V$. alginolyticus cells and lysed the host cells with a burst size of approximately 84 PFU/infected cell at an MOI of 1 . The Vp670 genome has a moderate size of 43,121 nucleotides coding for 49 ORFs and contains a two-component lysis module, which is composed of holA and $c w l Q$. holA is predicted to encode for the holin protein, HolA, and $c w l Q$ is predicted to encode for the endolysin protein, CwlQ. The expression of holA inside $E$. coli and $V$. alginolyticus cells exhibited strong toxicity to them, but the expression of $c w l Q$ alone inside $V$. alginolyticus cells did not generate obvious negative effects on the growth and structure of the cells. Both holA and $c w l Q$ likely play important roles during the release of progeny phages.

\section{Additional files}

Additional file 1: Table S1. The bacterial strains and plasmids used in this study. (DOCX $24 \mathrm{~kb}$ )

Additional file 2: Table S2. The accession numbers of DNA sequences of phages used for phylogenetic analysis in this study. (DOCX $30 \mathrm{~kb}$ )

Additional file 3: Table S3. The primers used in this study. (DOCX $24 \mathrm{~kb}$ )

Additional file 4: Figure S1. Phage plaques of Vp670 infecting $V$. alginolyticus E06333. (TIF $92 \mathrm{~kb}$ )

\section{Abbreviations}

DNAP: DNA polymerase; LB: Luria-Bertani; MOI: Multiplicity of Infection; OD: optical density; OM: outer membrane; ORF: Open Reading Frame; PFU: plaque forming units; PG: peptidoglycan; TEM: transmission electron microscopy

\section{Acknowledgements}

The authors would like to thank Guangzhou KnoGen Company Limited for their experimental cooperation and sample collection.

\section{Funding}

The research was supported by the Program of Fishery Problem Tackling of Guangdong Province (A201701B03), the Science and Technology Planning Project of Guangdong Province (2015B020231007 and 2017B030314052), and the Strategic Priority Research Program of the Chinese Academy of Sciences (XDA13000000). The funders had no role in the design of the study and collection, analysis, interpretation of data, nor in writing the manuscript.

\section{Availability of data and materials}

All of the datasets supporting the results of this article are included within the article and its additional files. Genomic sequence of phage Vp670 was deposited in GenBank (https://www.ncbi.nlm.nih.gov) under the accession number KY290756.

\section{Authors' contributions}

$\mathrm{PL}$ constructed the strains expressing HolA and $\mathrm{CW} \mid \mathrm{Q}$, analyzed the photos from TEM, and drafted the manuscript. LY isolated the phage and analyzed and adsorption rate of the phage. YYL analyzed one-step growth curve of the phage. YST tested the function of holA and $c$ WIQ. QTL performed the bioinformatic analysis of holA and CWIQ. WH and CQH conceived this study, reviewed, and gave critical revision of the manuscript. All authors read and approved the final manuscript.

\section{Ethics approval and consent to participate}

All experimental procedures and ethics approval were approved by the Department of Scientific Research and Planning of South China Sea Institute of Oceanology, Chinese Academy of Sciences.

\section{Consent for publication}

Not applicable.

\section{Competing interests}

The authors declare that they have no competing interests.

\section{Publisher's Note}

Springer Nature remains neutral with regard to jurisdictional claims in published maps and institutional affiliations.

\section{Author details}

${ }^{1}$ CAS Key Laboratory of Tropical Marine Bio-resources and Ecology (LMB), Guangdong Provincial Key Laboratory of Applied Marine Biology (LAMB), South China Sea Institute of Oceanology, Chinese Academy of Sciences, Guangzhou 501301, People's Republic of China. ${ }^{2}$ South China Sea Bio-Resource Exploitation and Utilization Collaborative Innovation Center, Guangzhou 510301, People's Republic of China. ${ }^{3}$ University of Chinese Academy of Sciences, Beijing 100049, China.

Received: 12 February 2018 Accepted: 28 September 2018 Published online: 11 October 2018

\section{References}

1. Zhang JC, Cao ZH, Xu YP, Li XY, Li HQ, Wu FF, Wang LL, Cao F, Li Z, Li SY, Li JJ. Complete genomic sequence of the Vibrio alginolyticus lytic bacteriophage PVA1. Arch Virol. 2014;159(12):3447-51.

2. Kim S, Aga DS. Potential ecological and human health impacts of antibiotics and antibiotic-resistant bacteria from wastewater treatment plants. J Toxicol Environ Health, Part B. 2007;10:559-73.

3. Silva YJ, Costa L, Pereira C, Mateus C, Cunha A, Calado R, Gomes NC, Pardo MA, Hernandez I, Almeida A. Phage therapy as an approach to prevent Vibrio anguillarum infections in fish larvae production. PLoS One. 2014;9(12): e114197.

4. Laganà P, Caruso G, Minutoli E, Zaccone R, Santi D. Susceptibility to antibiotics of Vibrio spp. and Photobacterium damsela ssp. piscicida strains isolated from Italian aquaculture farms. New Microbiol. 2011;34(1):53-63.

5. Tan D, Gram L, Middelboe M. Vibriophages and their interactions with the fish pathogen Vibrio anguillarum. Appl Environ Microbiol. 2014;80(10):3128-40.

6. Sime-Ngando T. Environmental bacteriophages: viruses of microbes in aquatic ecosystems. Front Microbiol. 2014;24(5):355.

7. Lv M, Wang S, Yan G, Sun C, Feng X, Gu J, Han W, Lei L. Genome sequencing and analysis of an Escherichia coli phage vB_EcoM-ep3 with a novel lysin, Lysep3. Virus Genes. 2015:50(3):487-97.

8. Merril CR, Biswas B, Carlton R, Jensen NC, Creed GJ, Zullo S, Adhya S. Longcirculating bacteriophage as antibacterial agents. Proc Natl Acad Sci U S A. 1996;93(8):3188-92.

9. Payne RJ, Phil D, Jansen VA. Phage therapy: the peculiar kinetics of selfreplicating pharmaceuticals. Clin Pharmacol Ther. 2000;68(3):225-30.

10. Oliveira H, Vilas Boas D, Mesnage S, Kluskens LD, Lavigne R, Sillankorva S, Secundo F, Azeredo J. Structural and enzymatic characterization of ABgp46, a novel phage endolysin with broad anti-gram-negative bacterial activity. Front Microbiol. 2016;7:208

11. Schmelcher M, Donovan DM, Loessner MJ. Bacteriophage endolysins as novel antimicrobials. Future Microbiol. 2012;7(10):1147-71.

12. Keary R, McAuliffe O, Ross RP, Hill C, O'Mahony J, Coffey A. Bacteriophages and their endolysins for control of pathogenic bacteria. In: Méndez-Vilas A, editor. Microbial pathogens and strategies for combating them: science, technology and education. Badajoz. Spain: Formatex Research Center; 2013. p. 1028-40.

13. Liu W, Lin YR, Lu MW, Sung PJ, Wang WH, Lin CS. Genome sequences characterizing five mutations in RNA polymerase and major capsid of phages $\varphi$ A318 and $\varphi$ As51 of Vibrio alginolyticus with different burst efficiencies. BMC Genomics. 2014;15(1):505.

14. Kalatzis $P G$, Bastías $R$, Kokkari $C$, Katharios P. Isolation and characterization of two lytic bacteriophages, $\varphi S t 2$ and $\varphi \mathrm{Grn} 1$; phage therapy application for biological control of Vibrio alginolyticus in aquaculture live feeds. PLoS One. 2016;11(3):e0151101. 
15. Carlson K. Working with bacteriophages: common techniques and methodological approaches. In: Elizabeth K, Alexander S, editors. Bacteriophages: biology and applications. Boca Raton: CRC Press; 2005. p. 428-84.

16. Li B, Zhang S, Long L, Huang S. Characterization and complete genome sequences of three N4-like roseobacter phages isolated from the South China Sea. Curr Microbiol. 2016;73(3):409-18.

17. Lin YR, Chiu CW, Chang FY, Lin CS. Characterization of a new phage, termed $\varphi$ A318, which is specific for Vibrio alginolyticus. Arch Virol. 2012; 57(5):917-26.

18. Bolger-Munro M, Cheung K, Fang A, Wang L. T4 bacteriophage average burst size varies with Escherichia coli B23 cell culture age. J Exp Microbiol Immunol. 2013;17:115-9.

19. Kropinski AM. Measurement of the rate of attachment of bacteriophage to cells. In: Clokie, MRJ, Kropinski AM, editors. Bacteriophages: methods and protocols (v1). Passaic: Humana Press; 2009.p.151-180.

20. Baudoux AC, Hendrix RW, Lander GC, Bailly X, Podell S, Paillard C, Johnson JE, Potter CS, Carragher B, Azam F. Genomic and functional analysis of Vibrio phage $\mathrm{SIO}-2$ reveals novel insights into ecology and evolution of marine siphoviruses. Environ Microbiol. 2002;14:2071-86.

21. Luo $P, H e X Y$, Liu QT, Hu CQ. Developing universal genetic tools for rapid and efficient deletion mutation in Vibrio species based on suicide T-vectors carrying a novel counterselectable marker, vmi480. PLoS One. 2015;10(12): e0144465

22. King AMQ, Lefkowitz E, Adams MJ, Carstens EB. Virus taxonomy: ninth report of the international committee on taxonomy of viruses. Amsterdam: Elsevier Incorporated; 2011.

23. Monier A, Claverie JM, Ogata H. Taxonomic distribution of large DNA viruses in the sea. Genome Biol. 2008;9(7):R106.

24. Daniels NA, Shafaie A. A review of pathogenic Vibrio infections for clinicians. Infect Med. 2000;17(10):665-85.

25. Sabir M, Ennaji Moulay M, Cohen N. Vibrio alginolyticus: an emerging pathogen of foodborne diseases. Int J Sci Technol. 2013;2:302-9.

26. Luo P, He XY, Wan YH, Liu QT, Hu CQ. Comparative genomic analysis of six newfound integrative conjugative elements (ICES) in Vibrio alginolyticus. BMC Microbiol. 2016;16:79

27. Lomelí-Ortega CO, Martínez-Díaz SF. Phage therapy against Vibrio parahaemolyticus infection in the whiteleg shrimp (Litopenaeus vannamei) larvae. Aquaculture. 2014;434:208-11.

28. Cerveny KE, DePaola A, Duckworth DH, Gulig PA. Phage therapy of local and systemic disease caused by Vibrio vulnificus in iron-dextran-treated mice. Infect Immu. 2002;70(11):6251-62.

29. Loessner MJ. Bacteriophage endolysins - current state of research and applications. Curr Opin Microbiol. 2005;8(4):480-7.

30. Gründling A, Manson MD, Young R. Holins kill without warning. Proc Natl Acad Sci U S A. 2001;98(16):9348-52.

31. Song J, Xia FF, Jiang HY, Li XW, Hu LY, Gong PJ, Lei LC, Feng X, Sun CJ, Gu JM, Han WY. Identification and characterization of HolGH15: the holin of Staphylococcus aureus bacteriophage GH15. J Gen Virol. 2016;97:1272-81.

32. Young R. Bacteriophage lysis: mechanism and regulation. Microbiol Rev. 1992;56(3):430-81.

33. Wang IN, Smith DL, Young R. Holins: the protein clocks of bacteriophage infections. Annu Rev Microbiol. 2000;54:799-825.

34. Young R. Phage lysis: three steps, three choices, one outcome. J Microbiol. 2014;52(3):243-58.

35. Bläsi U, Young R. Two beginnings for a single purpose: the dual-start holins in the regulation of phage lysis. Mol Microbiol. 1996;21:675-82.

36. Wang IN, Deaton J, Young R. Sizing the holin lesion with an endolysin-betagalactosidase fusion. J Bacteriol. 2003;185:779-7.

37. Garrett J, Bruno C, Young R. Lysis protein S of phage lambda functions in Saccharomyces cerevisiae. J Bacteriol. 1990;172:7275-7.

38. Shi Y, Li N, Yan Y, Wang H, Li Y, Lu C, Sun J. Combined antibacterial activity of phage lytic proteins holin and lysin from Streptococcus suis bacteriophage SMP. Curr Microbiol. 2012;65:28-34.

39. Drulis-Kawa Z, Majkowska-Skrobek G, Maciejewska B. Bacteriophages and phage-derived proteins-application approaches. Curr Med Chem. 2015;22:1757-73.

40. Saier MH, Jr RBL. Holins in bacteria, eukaryotes, and archaea: multifunctional xenologues with potential biotechnological and biomedical applications. J Bacteriol. 2015;197(1):7-17.
41. Cheng X, Zhang X, Pflugrath JW, Studier FW. The structure of bacteriophage T7 lysozyme, a zinc amidase and an inhibitor of T7 RNA polymerase. Proc Natl Acad Sci U S A. 1994;91(9):4034-8.

42. Briers Y, Volckaert G, Cornelissen A, Lagaert S, Michiels CW, Hertveldt K, Lavigne R. Muralytic activity and modular structure of the endolysins of Pseudomonas aeruginosa bacteriophages phiKZ and EL. Mol Microbiol. 2007; 65(5):1334-44.

\section{Ready to submit your research? Choose BMC and benefit from:}

- fast, convenient online submission

- thorough peer review by experienced researchers in your field

- rapid publication on acceptance

- support for research data, including large and complex data types

- gold Open Access which fosters wider collaboration and increased citations

- maximum visibility for your research: over $100 \mathrm{M}$ website views per year

At $\mathrm{BMC}$, research is always in progress.

Learn more biomedcentral.com/submissions 